\title{
Germanica
}

allemande au XXe siècle

\section{Ingeborg Bachmann : Undine geht. À la recherche d'un nouveau langage}

Undine geht. Eine Suche nach einer neuen Sprache.

\section{Marion Dufresne}

\section{OpenEdition}

\section{Journals}

Édition électronique

URL : http://journals.openedition.org/germanica/2074

DOI : 10.4000/germanica.2074

ISSN : 2107-0784

Éditeur

Université de Lille

Édition imprimée

Date de publication : 31 décembre 2002

Pagination : 113-127

ISBN : 9782913857100

ISSN : 0984-2632

Référence électronique

Marion Dufresne, «Ingeborg Bachmann : Undine geht. À la recherche d'un nouveau langage »,

Germanica [En ligne], 31 | 2002, mis en ligne le 19 mars 2013, consulté le 06 octobre 2020. URL : http:// journals.openedition.org/germanica/2074 ; DOI : https://doi.org/10.4000/germanica.2074

Ce document a été généré automatiquement le 6 octobre 2020.

(c) Tous droits réservés 


\title{
Ingeborg Bachmann : Undine geht. À la recherche d'un nouveau langage
}

Undine geht. Eine Suche nach einer neuen Sprache.

\author{
Marion Dufresne
}

1 Lorsqu'en 1961 Ingeborg Bachmann publie son recueil de nouvelles Das dreissigste Jahr ${ }^{1}$, la critique littéraire lui réserve un accueil peu enthousiaste. Marcel Reich-Ranicki, qui n'hésite pas à parler de la " poétesse déchue " ${ }^{2}$, n'est pas le seul à regretter qu'Ingeborg Bachmann, célèbre pour ses recueils de poésie Die gestundete Zeit et Anrufung des grossen Bären et reconnue par le prix littéraire que le groupe 47 lui a attribué en 1952, se soit tournée vers la prose. Dans le contexte agité des années 60 , l'auteur s'est vu reprocher l'absence de réflexion politique et une attitude de repli sur des thèmes intimistes ${ }^{3}$.

2 C'est seulement dans les années 80 que les échos positifs se multiplient. Le mouvement féministe, en particulier, accorde une importance croissante à l'œuvre en prose d'Ingeborg Bachmann, certains interprètes allant jusqu'à faire d'elle une de ses figures représentatives ${ }^{4}$.

3 Le texte Undine geht qui sera au cœur de notre propos, clôt le recueil Das dreissigste Jahr qui comporte six nouvelles. Déconcertant par la spécificité de sa forme qui interdit une classification aisée en tant que genre clairement défini, son titre induit en erreur. Il annonce un récit à la troisième personne. Or, il s'agit d'une longue plainte, d'un reproche amer prononcé à la première personne et qui commence par un véritable cri : « Hommes! Monstres! »5. On a souligné à juste titre que, en vertu de sa thématique, ce texte constitue un pont entre la pièce radiophonique Der liebe Gott von Manhattan et le projet Todesarten que l'auteur n'eut pas l'occasion de mener à terme et dont font partie les deux romans Malina et Der Fall Franza (Le cas Franza), restés, eux aussi, inachevés.

4 Bien qu'il convienne de nuancer certains jugements quelque peu réducteurs, ce texte formule effectivement l'interrogation centrale de l'œuvre d'Ingeborg Bachmann: comment reconstruire un langage commun, affranchi du désir omniprésent de domination et qui seul pourrait permettre de rompre, au moins momentanément, la profonde solitude de l'homme. S'il semble justifié d'affirmer qu'en ce sens Undine geht 
aborde un sujet typiquement bachmannien, ce texte se distingue du reste de sa production par deux particularités qui sont, d'une part sa forme très spécifique, d'autre part la référence explicite, annoncée dans le titre même, à des modèles littéraires existants.

Dans cette étude nous aborderons ce dernier aspect, celui de la réécriture. Il s'agit d'un phénomène très courant, mais toujours d'un intérêt particulier dans la mesure où ce choix traduit la volonté de transmettre, sous une forme et d'une manière qui lui est propre, un contenu précis ayant subi un remaniement significatif de la part de l'auteur. Dans le cas précis de Undine geht, la critique a établi le lien entre le conte Undine du poète romantique Friedrich de La Motte-Fouqué et de l'Ondine de l'auteur français Jean Giraudoux ${ }^{6}$. On a également évoqué d'autres influences possibles, notamment celle du compositeur allemand Henze avec qui, depuis 1953, Bachmann partageait un logement sur l'île d'Ischia. En 1957, Henze créa un opéra intitulé Undine ${ }^{7}$. La recherche la plus complète dans ce domaine a été menée par Mona Le Nawab qui, en 1993, a publié une étude comparative intitulée Ingeborg Bachmanns 'Undine geht': ein stoff-und motivgeschichtlicher Vergleich mit Friedrich de La Motte-Fouqués 'Undine' und Jean Giradoux' 'Ondine's.

6 Il ne peut s'agir pour nous de fournir une autre comparaison entre ces trois textes bien qu'il soit évidemment nécessaire de se référer à ces modèles afin de mieux saisir la spécificité de la nouvelle bachmannienne. Il convient dans un premier temps de s'interroger sur les raisons qui ont amené l'écrivain autrichien à reprendre un sujet faisant partie du patrimoine culturel européen ayant inspiré plusieurs auteurs à des périodes historiques différentes. Le texte d'Ingeborg Bachmann porte les traces d'une connaissance approfondie de ces ouvrages ce qui nous incitera à examiner plus précisément ses références à ses prédécesseurs et à étudier l'usage qui est fait de ces modèles. Cette analyse devrait permettre de dégager la problématique propre d'Undine geht et de définir plus clairement l'objectif d'Ingeborg Bachmann. La quête désespérée, mais jamais abandonnée d'un langage nouveau permettant une véritable communication entre les hommes constitue sans aucun doute une constante de son œuvre.

7 En 1959, Ingeborg Bachmann inaugure la chaire de poésie qui vient d'être créée par l'université de Francfort par un cycle de conférences intitulé Probleme zeitgenössischer Dichtung (Problèmes de la poésie contemporaine). Abordant lors de la quatrième conférence «la fréquentation des noms » (über den Umgang mit Namen), l'auteur évoque celui d' Undine en le désignant comme un nom qui «s'ancre pour toujours dans la conscience avec son aura $»^{9}$. En choisissant le nom d'Undine pour son personnage principal, elle souhaite faire appel à l'imaginaire collectif et susciter par là même des attentes précises de la part du lecteur. Undine est d'abord un personnage né de l'imagination des poètes. Pour celui qui partage l'héritage culturel d'Ingeborg Bachmann, la simple évocation de ce nom suffit pour que naisse devant ses yeux un être féminin, une créature de l'eau, appartenant au domaine surnaturel. Dans son Liber de nymphis, sylphis, pygmalis et salamandris et de ceteris spiritibus ${ }^{10}$, Paracelse dont s'inspirait toute l'école romantique, définit les ondines comme des êtres ressemblants physiquement aux hommes, mais privés d'âme. C'est exclusivement en épousant un humain qu'une ondine peut en recevoir une. Paracelse ne manque pas de mentionner le risque lié à une telle union : l'ondine est condamnée à donner la mort à l'homme infidèle, ce qui signifie que ce dernier s'engage pour toujours. 
8 L'ondine des contes représente « le principe féminin », incarne l'idée de la femme à la fois enfant de la nature et séductrice dangereuse. Femme-enfant insoucieuse, ignorant les règles les plus élémentaires de la morale et de la bienséance, elle s'offre à l'homme sans aucune pudeur ni retenue. Belle et mystérieuse, elle fait preuve d'une gentillesse et d'une soumission sans pareil. Seulement, le lecteur sait cette rencontre amoureuse condamnée à l'échec : liant son destin à celui de l'homme, l'ondine sera inévitablement trahie. Le titre choisi par Bachmann indique que l'histoire d'Undine sera reprise après sa rupture avec le monde des humains, mais signale aussi un changement de perspective important. Undine geht suggère un rôle actif du personnage féminin qui semble prendre de son plein gré la décision de partir. La curiosité du lecteur se trouve éveillée, son attente devient autre.

9 La suite du texte confirme la première impression : cette Undine possède une forte personnalité et fait preuve de détermination. C'est elle qui s'adresse aux hommes, « ces monstres ». Dans un long monologue chargé d'une agressivité qui ne parvient pas à dissimuler une douleur profonde, elle reproche à Hans son immense lâcheté qui le fait renoncer à tous ses grands projets au profit d'une vie dans la sécurité matérielle, certes, mais ennuyeuse et répétitive.

Les interprètes sont nombreux à signaler que le prénom Hans (qui était Huldbrand pour le personnage masculin principal dans le conte de La Motte-Fouqué et qui devient « Jean » chez Giraudoux) est non seulement un prénom très répandu, mais qu'il désigne traditionnellement l'homme ordinaire, l'homme issu du peuple, celui que l'on rencontre dans la vie de tous les jours et qui ne se distingue en rien de ses semblables. Ingeborg Bachmann mêle, nous y reviendrons, habilement les références à un être singulier, un individu portant le nom de Hans et aux hommes en général.

11 Hans et Undine perdent en individualité ce qu'ils gagnent en représentativité. C'est pourquoi l'usage de leurs noms permet une entrée directe en matière : nul besoin de s'attarder sur la psychologie des personnages, ni de justifier leur apparition dans un certain lieu, dans une situation donnée. Il paraît évident que l'auteur, au moyen de la réécriture d'un modèle célèbre, supposé connu, cherche à établir un contact direct avec le lecteur en instaurant une sorte de jeu fait d'attentes implicites tantôt satisfaites, tantôt déçues.

12 Déjà les premières lignes d'Undine geht suffisent pour désorienter le lecteur : le récit, rédigé contre l'attente suscitée par le titre, à la première personne, commence par une exclamation violente. "Hommes! Monstres! Hans, nom de ces monstres, nom que je n'oublierai jamais! ${ }^{11}(209)$. Très probablement ce sont là les paroles d'Undine (son nom n'apparaît en effet que dans le titre), mais il est impossible de déterminer avec certitude si elle s'adresse à un individu masculin en particulier ou au genre humain en général. Cette ambiguïté se révèle constitutive pour le texte entier, car, tout au long de son monologue, Undine passe, sans transition aucune, du «tu » au "vous », de Hans, être unique et différent de tous les autres, à tous les Hans qu'elle a aimés.

La rencontre amoureuse, dont il est question ici, constitue un événement à la fois singulier et habituel, nous sommes, comme le fait remarquer Peter von Matt, confronté à un phénomène paradoxal : "l'événement singulier se répète ${ }^{12}$. " Par conséquent, le texte se caractérise par un va-et-vient perpétuel entre le présent et le passé, entre l'action précise du moment et les événements du passé qui ressemblent à des actes rituels. La plainte d'Undine se place entièrement sous le signe de cette contradiction 
interne, d'où un mouvement dont la direction se trouve continuellement inversée pour donner finalement l'impression d'une évolution en spirale.

Afin de rendre compte de cette particularité sur laquelle se fonde, selon nous, le monologue d'Undine, nous proposons une analyse du texte qui met en relief les multiples tensions sur lesquelles repose sa force de conviction, mais dans lesquelles il faut également chercher l'origine d'un certain désarroi face à une pensée qui refuse les limites pour tendre vers l'utopie.

En optant en faveur d'un nom " auratique », Ingeborg Bachmann suscite, nous l'avons souligné, des attentes précises du lecteur. De plus, elle manifeste par ce choix sa volonté d'insister sur l'universalité de ses propos. Ainsi l'union entre le personnage mythique d'Undine et son partenaire Hans reste certes au centre d'une histoire unique, néanmoins il s'agit davantage encore de toute relation pouvant s'établir entre un être humain et une figure représentative d'un monde régi par d'autres lois. Bien que limitée dans le temps et, selon l'auteur, bien que condamnée à l'échec, elle se réalisera toujours à nouveau. Irrésistiblement attirés l'un par l'autre, Hans et Undine ne cesseront de s'appeler et se consumer dans la nostalgie d'une impossible fusion. Dans leur tentative de définir qui ou ce qui est désigné sous le nom d'Undine, les commentaires critiques sont nombreux à affirmer que, si elle représente le principe féminin, l'« anima » à la recherche du partenaire masculin idéal, Undine symbolise également l'aspiration profonde de l'homme à trouver l'être complémentaire, celui qui seul saurait lui permettre de se réaliser pleinement ${ }^{13}$. En mettant en garde contre une interprétation qui identifierait l'auteur au personnage d'Undine, Ingeborg Bachmann livre une clé importante pour la compréhension de la nouvelle et ses intentions :

Les lecteurs, et les auditeurs, identifient immédiatement le Je à l'auteur puisque la nouvelle est écrite à la première personne. Ce n'est pas du tout le cas. Ondine n'est pas une femme, ni même un être vivant, mais, pour reprendre les mots de Büchner, «l'Art, ah, l'Art». C'est de l'autre côté qu'il faut chercher l'auteur, moi en l'occurrence, parmi ceux qui s'appellent Hans ${ }^{14}$.

16 Avant de cerner le rôle attribué à Undine, il est utile de s'interroger sur l'identité de Hans et de préciser ses attentes. Selon le commentaire de l'auteur, Hans désigne l'espèce humaine, sans distinction entre les sexes, avec néanmoins une précision importante. Hans est l'homme en quête d'absolu, celui qui ne parvient pas à se contenter définitivement de son sort. Nous retrouvons chez lui des attitudes du «Jean » de la pièce de théâtre de Giraudoux. Ce chevalier demande la main d'Ondine à son père adoptif et, pour justifier son geste, s'exclame :

Nous découvrions des palais et nous revenions habiter nos manoirs. Nous délivrions Andromède et cela nous valait le droit à une retraite à soixante ans. Nous ravissions le trésor des géants et cela nous donnait la dispense du maigre les vendredis... (...) Il était un chevalier qui cherchait dans ce monde ce qui n'est pas usé, quotidien, éculé ${ }^{15}$.

17 Et à Ondine Jean confie : «Depuis mon enfance, un hameçon m'arrachait à ma chaise, à ma barque, à mon cheval... tu me tirais à toi... $»^{16}$. Le Hans de Bachmann aussi semble vivre en sursis, il tente de se construire tout un réseau de certitudes, mais son équilibre reste fragile. Il n'a jamais vraiment abandonné l'idée d'une fuite, d'une existence radicalement autre hors de toute convention sociale, sans aucune sécurité matérielle. C'est ce désir secret qui le rend apte à entendre l'appel d'Undine et c'est ce qui le distingue des femmes qui, elles, s'accommodent d'une vie somme toute banale, sans surprises et sans risques ${ }^{17}$. 
Mais les hommes sont tout silence. Ils caressent affectueusement les cheveux de leurs femmes et de leurs enfants, ouvrent en grand la radio, ce qui ne les empêche pas d'entendre le bruit de la mer dans le coquillage, la fanfare du vent. Plus tard, lorsqu'il fait sombre dans les maisons, ils se lèvent à la dérobée, entrouvrent la porte, pénètrent dans le couloir sur la pointe des pieds, descendent dans le jardin, enfilent une allée et alors, soudain, distinctement, ils perçoivent le cri de souffrance, l'appel venu de loin, la musique fantomatique. Viens! Viens! Viens donc enfin !18 (211). ambigu. Undine dit avoir aimé les hommes parce qu'ils n'ont pas résisté à son appel,
mais elle précise aussi que ce sont les hommes qui l'ont appelée en rêvant d'elle, de l'«autre $»^{20}(214,217)$. Si Undine n'est donc pas une femme, elle serait plutôt l'incarnation d'un désir inconscient, une illusion née de l'imagination. C'est ainsi qu'Auguste, le père adoptif d'Ondine de Giraudoux définit cette dernière : "Quand elle est partie, tout d'elle est parti. Quand elle est partie, elle n'est jamais venue. C'est un rêve, Ondine. Il n'y a pas d'Ondine $»^{21}$. Undine, multiforme, toujours la même et toujours une autre est une projection de tout ce que l'homme voudrait qu'elle soit, également de tout ce qu'il aimerait être, lui. Cette aspiration, très importante déjà pour caractériser Jean dans la pièce de Giraudoux, devient un élément moteur dans la nouvelle d'Ingeborg Bachmann. Quant à Undine, elle reste insaisissable. Elle semble faire un avec l'élément dont elle est issue. L'eau, symbole de l'inconscient ${ }^{22}$, est présente sous toutes ses formes, il est question de "gouttes», de "grandes eaux», de «pluie», de « fleuves » et de « mers », de « l'eau du Danube » et « du Rhin », du « Tibre », du « Nil », «l'océan Arctique », des « eaux d'encre de la haute mer », des « marais ensorcelants », d'« eau dans mes mains ». Sur la dizaine de pages que compte le monologue, le terme « eau » apparaît 18 fois et avec des connotations multiples. C'est l'élément naturel indispensable à tout être vivant, fascinant par sa beauté et sa diversité. Néanmoins, l'homme craint cet élément qu'il cherche à dompter, mais qui lui échappe malgré ses efforts et qui devient alors un réel danger. Attiré par l'eau qui lui renvoie le reflet de son propre visage (nous retrouvons ici le mythe de Narcisse), l'homme risque de perdre son équilibre et le contrôle de ses émotions. Le tableau final de la nouvelle nous présente un homme (Hans?) au bord de l'abîme

Je suis sous les eaux. Sous les ondes.

Et là-haut quelqu'un avance, qui déteste les eaux et qui déteste les feuillages, quelqu'un qui ne comprend pas, qui ne comprendra jamais. Comme moi je n'ai jamais compris ${ }^{23}$. (220)

L'auteur renvoie ici à un autre modèle connu, au poème Der Fischer (Le Pêcheur) de Goethe (1778) qui repose sur cette même ambiguïé : le pêcheur se noie dans les flots en cédant à l'appel de l'eau, mais aussi en obéissant à une voix intérieure. Hans, le pêcheur, Huldbrand, Jean - leur histoire d'amour est intemporelle et ne connaît pas de fin, et est pourtant limitée «à un long moment [où] on ne vous comprenait pas, vous ne saviez plus rien, $(. . .)^{24} »(214)$. Elle a pour arrière plan un paysage bien précis et se déroule n'importe où : près du Danube, en Forêt Noire ou sur un boulevard (215). Cette union s'achèvera dans une gare, avant minuit, selon une loi qui, indépendamment de la volonté de l'homme, s'accomplira infailliblement : la solitude de l'un n'annulera jamais celle de l'autre, leurs mondes ne se fondront jamais en un seul. 
21 C'est pourquoi la nouvelle de Bachmann se construit autour du mot clé de la trahison. Celle-ci est effectivement double ${ }^{25}$ : pour rejoindre Undine, Hans est obligé d'abandonner femme et enfants. Face à Undine, il va même jusqu'à comparer sa vie conjugale à un enfer et semble se plaire dans le rôle de celui qui se sacrifie entièrement pour les autres (212). Mais inévitablement arrivera le moment des grands regrets et l'homme se mettra à trahir une deuxième fois. Undine est abandonnée à son tour et Hans regagne son foyer. Il ne paie pas cette trahison de sa vie, puisqu'Undine, si elle est, comme les héroünes de La Motte-Fouqué et de Giraudoux, condamnée à retourner dans l'eau, n'a aucune obligation de tuer son amant infidèle. On peut se poser la question si ce retour à « la vie normale » ne constitue pas pour ce dernier une punition plus grande encore. En une allusion directe au modèle allemand, Bachmann désigne Undine comme « la pleureuse lors de vos noces, surgissant les pieds mouillés, et sous mon baiser vous redoutez la $\operatorname{mort}^{26} \gg(217)$.

Mais cette ultime délivrance n'aura pas lieu, l'homme est obligé de vivre avec ses regrets au même titre qu'Undine est condamnée à se souvenir, à ne rien pouvoir oublier. C'est ce qui la distingue fondamentalement de l'Ondine de Giraudoux qui, au troisième appel des ondines, va perdre la mémoire ${ }^{27}$. «Ma mémoire est inhumaine. Trahison, bassesse, ma mémoire vous garde ${ }^{28} »(217)$.

L'Undine d'Ingeborg Bachmann en revanche n'aurait pas de raison d'exister si elle cherchait, à l'instar du personnage soumis et humble de La Motte-Fouqué et de celui, affranchi et désinvolte de l'auteur français, à obtenir son intégration dans le monde des humains. Paradoxalement, c'est son refus de ce dernier qui réhabilite l'homme :

Comment croirais-je à vos dilemmes? Comment croire, aussi longtemps que j'ai foi en vous, que vous valez plus que vos vains propos, vos actions pitoyables, vos folles suspicions ? ${ }^{29}$ (216)

Dans la nouvelle de Bachmann, c'est à l'homme de prendre la décision de rompre avec ses habitudes, de briser les chaînes d'une existence déterminée par les conventions. C'est lui qui accepte de renoncer à penser en termes d'utilité et de rendement, à perdre ses repères sécurisants. C'est seulement lorsqu'il se met dans cette position d'apparente faiblesse qu'Undine peut venir à lui pour servir d'intermédiaire entre l'homme et la connaissance, lui révéler la vérité. Inversant ainsi les rapports de force entre les deux partenaires et en modifiant radicalement l'échelle des valeurs, Bachmann rompt avec le schéma en vigueur chez l'auteur allemand de l'époque romantique et repris en partie par Giraudoux, afin de renouer avec une tradition plus ancienne écartée au profit de la religion chrétienne. Ainsi Claude Lecouteux démontre que, à l'origine, l'homme aspirait à une union avec l'ondine dans l'espoir de partager ses pouvoirs surnaturels et d'acquérir l'immortalitée ${ }^{30}$.

C'est précisément ce qu'Undine propose à son partenaire. Undine possède le pouvoir de défier les lois de la temporalité, grâce à elle, l'homme saura vivre pleinement l'instant, connaîtra une existence qui semble suspendue tant que dure son union avec Undine. «Moi, je vous ai livré mon enseignement d'un regard. Tout était alors parfait, clair et fou. Je vous ai dit : la mort est là. Et : temps, suspends ton cours ! $»^{31}(215)$. Ces moments sont précieux, mais éphémères. Ce sont, si l'on revient au commentaire précité d'Ingeborg Bachmann, des instants de créativité poétique qui, peut-être, verront naître l'œuvre d'art. Fruit de leur rencontre, cette dernière matérialise leur union. Seulement cet état de grâce ne saurait durer, il reste l'exception, le but inaccessible, celui dont l'auteur parle dans son désormais célèbre discours à l'occasion de la remise du prix 
attribué par l'association des aveugles de guerre pour la meilleure pièce radiophonique en 1959. Juste après avoir précisé qu'elle était parfaitement consciente du fait que vivre en marge de la société n'est pas une solution envisageable, elle affirme qu' « à l'intérieur des limites, nous tendons vers ce qui est parfait, impossible et inaccessible, que ce soit en amour, dans le domaine de la liberté ou dans tout autre dimension absolue ${ }^{32}$.»

Le poids de l'œuvre d'art se mesure moins en termes de résultats qu'en tant qu'en termes d'effort permanent pour s'approcher d'un but qui n'en finit pas de s'éloigner. L'art, qui est recherche d'un idéal, est aussi indispensable à l'homme que réciproquement, l'un ne saurait exister sans l'autre ${ }^{33}$. Au même titre que l'œuvre d'art possédant une vie qui lui est propre, Undine n'est pas exclusivement le pur produit de l'imagination. La définir comme la force inconsciente qui habite l'homme, le principe féminin ${ }^{34}$, est certes justifié. Le texte de Bachmann suggère l'identité de Hans et Undine lorsque cette dernière désigne l'élément auquel elle appartient comme la «frontière humide entre moi-même et moi-même $»^{35}(210)$.

Sans aucun doute elle incarne aussi le rêve d'une existence totalement autre, en dehors des conventions sociales et libre de toutes contraintes. Elle est «l'autre », ce contraire, cette nostalgie indéfinie sans laquelle l'homme ne sera jamais l'être entier, "complet ", auquel il aspire. Et c'est paradoxalement en ayant recours à des attributs qui mettent l'accent sur son caractère insaisissable qu'Ingeborg Bachmann parvient à donner des contours concrets à son personnage. Nous partageons l'avis de Dagmar Kann-Coomann pour qui Undine est la personnification allégorique de l'art. Sa relation avec Hans reflète, selon l'interprète, le rapport qu'entretient l'auteur avec l'art ${ }^{36}$. Celui-ci requiert l'homme entier et ne tolère aucun compromis. La situation de l'écrivain est effectivement celle de Hans. Le poète est constamment tiraillé entre le besoin fondamental de se consacrer à son art et les exigences de la vie quotidienne. Éternellement insatisfait et souffrant de son déchirement intérieur, il ne trouvera son salut que le temps de la création et le remettra en jeu à chaque fois que son œuvre s'émancipe pour mener son existence autonome. Il va de soi que contrairement au conte allemand et à la pièce de Giraudoux (dans les deux cas, l'ondine est une jeune fille d'une beauté éclatante, aux traits fins et à la silhouette svelte), le texte ne contient aucune description de son physique. Elle ne séduit pas non plus par un don particulier, ce n'est ni la Lorelei du poème de Heine qui, outre ses longs cheveux dorés (signe de féminité par excellence), possède également une voix magnifique, ni même la sirène malheureuse qui sacrifie cette voix d'abord, sa vie ensuite et dont Andersen a fait l'héroïne de son célèbre conte Den lille Havfrue (La Petite sirène) ${ }^{37}$. Néanmoins, Undine n'est pas muette, elle aussi attire l'homme à l'aide de sa voix. Hans ne saurait résister à cet appel qu'Undine renouvelle presque malgré elle, «l'appel qui signifiait la fin de tout $^{38} »(214)$.

La rencontre amoureuse est entièrement placée sous le signe de la parole, on est frappé par l'abondance des verbes «sprechen» (parler), «reden» (dialoguer) et surtout "sagen" (dire). Les actes de parole sont répartis de façon parfaitement équilibrée entre les deux protagonistes, leur échange verbal intense reflète leurs efforts pour se rapprocher l'un de l'autre. La distance les séparant est considérable, c'est elle qui est au centre du seul dialogue repérable par la forme extérieure du texte et qui semble depuis toujours être au commencement de leur relation :

«Bonsoir.

Bonsoir.

Y a-t-il loin jusqu'à toi? 
Loin, loin.

Loin jusqu'à moi aussi. ${ }^{39}(211)$ soin de présenter Ondine à plusieurs reprises dans le rôle de la dompteuse des eaux tandis que l'homme est à leur merci. Cependant, le personnage masculin d'Ingeborg Bachmann, quant à lui, sait se protéger contre l'emprise du monde représenté par Undine. Il s'empresse de le désavouer publiquement pour rétablir sa sécurité, pour retrouver ses certitudes. Hans et Undine appartiendront toujours à deux mondes différents dont les lois ne peuvent se transgresser que par intermittence. Au même titre qu'Undine est condamnée à retourner dans son élément, la condition d'humain de Hans fait qu'il ne pourra jamais quitter définitivement l'univers qui est le sien. Ce dernier est hors de la portée d'Undine qui refuse catégoriquement de trahir sa loi : c'est paradoxal - mais il faudrait dire logique, si l'on adopte le point de vue d'Undine - au moment où elle peut dire à Hans: «Nous nous aimions. Notre génie était le même.» (215), elle avoue également: "Je ne te comprends pas, ne comprends pas, ne puis comprendre $»(214)^{42}$.

31 Jamais elle ne pourra partager les soucis de Hans, ayant trop bien compris la « subtile politique, ces idées, ces opinions, ça et le reste (...), ces conférences, vos menaces, vos preuves, vos faux semblants, (...) il n'y avait plus rien à comprendre ${ }^{43} »(214)$.

À travers Hans et Undine ce sont deux attitudes fondées sur des priorités radicalement opposées qui s'affrontent. Dès le début de son monologue, Undine annonce les conditions de sa vie « après Hans » qui la condamne au mutisme en évoquant son retour à l'eau parmi tous ces êtres eux aussi privés du langage (210). On peut établir un parallèle intéressant avec le conte précité d'Andersen. Comme Undine, la petite sirène a pu vivre des moments heureux en compagnie du prince, mais étant privée de sa langue (c'était le prix qu'elle a dû payer pour obtenir des jambes à la place de sa queue de poisson) et par conséquent de la parole, elle ne réussit pas à faire comprendre au prince que c'est elle qui l'avait secouru après son naufrage dans la mer déchaînée. Seul le dialogue aurait permis la compréhension mutuelle et aurait rétabli la vérité. Mais le prince pense avoir reconnu en la fille du roi celle qui lui a sauvé la vie et en l'épousant, condamne la sirène à la mort.

33 La nécessité d'un langage commun est prépondérante et c'est précisément ce dernier qui s'effrite. Comme pour faire écho à ce silence, la dernière partie de sa plainte attribue l'acte de parole exclusivement à Hans, mais la maîtrise parfaite qu'il en possédait, appartient au passé. On relève, sur la dernière page de la nouvelle, pas moins de onze fois les verbes sprechen, sagen, reden et cinq fois les mots Reden et Worte. Comme 
Undine commande aux vagues, Hans, lui, régnait en maître sur les paroles. Seulement, sans le secours d'Undine, l'homme n'a pas accès à toute la vérité et doit se contenter d'une vérité qui pourrait être meurtrière, mais qui reste une vérité partielle ${ }^{44}(220)$. D'où la nécessité de tout recommencer dès le début (et de refaire les mêmes erreurs inéluctables). C'est pourquoi nous considérons les paroles ultimes du texte comme une dernière - et première - situation de rencontre entre Hans et Undine qui, ayant perdu leur langage commun, perçoivent malgré tout l'appel désespéré de l'autre :

Presque réduit au silence,

percevant au loin

encore

l'appel.

Viens. Une fois seulement.

Viens. $(220)^{45}$

La nouvelle s'achève selon nous sur une note d'espoir. La séparation de Hans et d'Undine ne semble pas définitive, même si elle se reproduira. Elle fait partie d'une loi secrète à laquelle nul ne saurait échapper. La séparation reste inéluctable, mais aussi temporaire que l'union. Les dernières paroles prononcées sont une invitation adressée à l'autre, formulée dans l'espoir d'être entendue malgré la distance. Contrairement à ses célèbres modèles, Ingeborg Bachmann a choisi une fin plus ouverte, moins définitive. Non seulement Hans ne meurt point, mais l'adieu d'Undine semble également révocable. Viendra le moment de la nuit tombante où elle percevra l'appel de Hans et, obéissant à sa loi intérieure, ira à la rencontre de l'homme. L'auteur, dont les deux romans sont restés inachevés, connaît trop bien le déchirement qui est celui de Hans. Elle partage avec lui le besoin d'un cadre de vie dont les règles procurent un minimum de stabilité tout en éprouvant cette indéfinissable envie de renoncer à toutes les certitudes. Tout porte à croire que, jusqu'à sa mort tragique, Ingeborg Bachmann n'a jamais abandonné sa conviction que l'artiste a un rôle social et que sa réconciliation avec le monde qui l'entoure n'est pas condamnée à rester une utopie. Elle passera obligatoirement par le langage de vérité que nous enseigne l'œuvre d'art, car «le poète peut réussir deux choses : représenter, représenter son époque et présenter quelque chose dont le temps n'est pas encore venu $»^{46}$.

\section{NOTES}

1. Dans les notes, nous citerons Undine geht d'Ingeborg Bachmann dans l'édition Piper en quatre volumes publiée par Christine Koschel, Inge von Weidenbaum et Clemens Münster. Ingeborg Bachmann, Werke. Zweiter Band : Erzählungen, München/Zürich, R. Piper \& Co. Verlag, 1984.

2. Reich-Ranicki, Marcel, Eine einst bedeutende Lyrikerin auf sonderbaren Abwegen. Am liebsten beim Friseur. Ingeborg Bachmanns neuer Erzählband 'Simultan' in : Die Zeit, 29. September 1962.

3. Voir à titre d'exemple: Wondratschek, Wolf, Die utopische Idee. Zur Prosa Ingeborg Bachmanns in : Text und Kritik 6, 1964, S.8-12.

4. C'est le cas de Holger Pausch, Ingeborg Bachmann. Köpfe des 20. Jahrhunderts, Berlin, Colloquium Verlag, 1975, mais également de Peter Horst Neumann, Vier Gründe einer Befangenheit. Über 
Ingeborg Bachmann in : Merkur 32, 1978, S.1130-1136 et Christa Gürtler, Schreiben Frauen anders? Untersuchungen zu Ingeborg Bachmann und Barbara Frischmuth, Stuttgart, 1983.

5. Pour la traduction française nous nous référons à l'ouvrage de Marie-Simone Rollin, L'adieu de la sirène Ondine dans: La trentième année. Nouvelles, Paris, Édition du Seuil, 1964, p. 207-220. Les indications entre parenthèses dans le texte renvoient à cette pagination.

6. La Motte-Fouqué, Friedrich de, Undine dans : Werke, Hrsg. Walther Ziesemer, Hildesheim, New York, Goldene Klassiker Bibliothek, 1973. Giraudoux, Jean, Ondine. Pièce en trois actes 1939, d'après le conte de Frédéric de La Motte- Fouqué, Paris, Librairie Générale Française, 1990.

7. Hans-Werner Henze, Undine. Tagebuch eines Ballets, München, Piper, 1959.

8. Mona Le Nawab, Ingeborg Bachmanns « Undine geht» : ein stoff- und motivgeschichtlicher Vergleich mit Friedrich de La Motte-Fouques « Undine » und Jean Giraudoux” " Ondine ». Würzburg, Königshausen und Neumann, 1993.

9. Ingeborg Bachmann, Frankfurter Vorlesungen dans: Werke. Vierter Band, München/ Zürich, R. Piper \& Co. Verlag, 1984. Pour la traduction française, nous nous référons à l'ouvrage d'Elfie Poulain, Ingeborg Bachmann, Leçons de Francfort. Problèmes de poésie contemporaine, Arles, Éditions Actes Sud, 1986.

10. Paracelse, Liber de nymphis, sylphis, pygmalis et salamandris et de ceteris spiritibus in : Sämtliche Werke, Band 1, Hrsg. Karl Sudhoff und Wilhelm Malthiessen, Hildesheim, Georg Olms, 1996.

11. Undine geht, p. 253 : «Ihr Menschen! Ihr Ungeheuer! Ihr Ungeheuer mit Namen Hans! Mit diesem Namen, den ich nie vergessen kann. »

12. Von Matt, Peter, Liebesverrat. Die Treulosen in der Literatur, München, Wien, Carl Hanser, 1989, p. 243.

13. Citons à titre d'exemple l'article de Schuscheng, Dorothe, Arbeit am Mythos Frau. Weibliche Autonomie und Geschlechterhierarchie: Ingeborg Bachmanns Rezeption des biblischen Gomorrha-Mythos und des aus Märchen und Mythen stammenden Undine-Motivs. In : Arbeit am Mythos Frau. Weiblichkeit und Autonomie in der literarischen Mythenrezeption Ingeborg Bachmanns, Christa Wolfs und Gertrud Leuteneggers. Frankfurt/M. /Bern u.a., Peter Lang, 1987, (europäische Hochschulschriften, Reihe I : Deutsche Sprache und Literatur, Band 1006), p. 22.

14. Nous avons repris la traduction d'Anne-Sophie Astrup dans: L'enjeu du Je dans: Ingeborg Bachmann Cahiers du Grif Paris, Édition Tierce, 1987, p. 11. S. 46 : «Denn die Leser und auch die Hörer identifizieren ja sofort - die Erzählung ist ja in der Ich-Form geschrieben - dieses Ich mit dem Autor. Das ist aber keineswegs so. Die Undine ist keine Frau, auch kein Lebewesen, sondern, um es mit Büchner zu sagen, 'die Kunst, ach, die Kunst'. Und der Autor, in dem Fall ich, ist auf der anderen Seite zu suchen, also unter denen, die Hans genannt werden. »

Interview avec Ingeborg Bachmann du 5.11.1964 publié dans : Ingeborg Bachmann. Gespräche und Interviews. München, Piper \& Co., 1983 'Wir müssen wahre Sätze finden',

15. Ondine, p. 39.

16. Ibid., p. 47.

17. Nous partageons le jugement de von Matt qui affirme qu'Undine ne prend en aucun cas position en faveur des femmes humaines. Liebesverrat. Die Treulosen in der Literatur, p. 248.

18. Undine geht, p. 257 : «Aber die Männer schweigen dazu. Fahren ihren Frauen, ihren Kindern treulich übers Haar, schlagen die Zeitung auf, sehen die Rechnungen durch oder drehen das Radio laut auf und hören doch den Muschelton, die Windfanfare, und dann noch einmal, wenn es dunkel ist in den Häusern, erheben sie sich heimlich, öffnen die Tür, lauschen den Gang hinunter, in den Garten, die Alleen hinunter, und nun hören sie es ganz deutlich : Den Schmerzton, den Ruf von weither, die geisterhafte Musik. Komm ! Komm ! Nur einmal komm ! »

19. Ibid., p. 256 : « (...), denn es wäre nichts als das Eingeständnis, dass man noch mehr als durch alles andere verführbar ist durch einen Schmerzton, den Klang, die Lockung und ihn ersehnt, den grossen Verrat. » 
20. Ibid., p. 257 : «Ihr Ungeheuer, dafür habe ich euch geliebt, dass ihr wusstet, was der Ruf bedeutet, dass ihr euch rufen liesst... » et p. 260 « Doch vergesst nicht, dass ihr mich gerufen habt in die Welt, dass euch geträumt hat von mir, der anderen, dem anderen... »

21. Ondine, p. 42.

22. Pour la symbolique de l'eau, voir par exemple Carl Gustav Jung, Gestaltung des Unbewussten, Zürich, Rascher, 1960.

23. Ibid., p. 263 : « Und nun geht einer oben und hasst Wasser und hasst Grün und versteht nicht, wird nie verstehen. Wie ich nie verstanden habe. »

24. Ibid., p. 257 : «Das währte eine herrliche und grosse Weile lang, dass ihr nicht verstanden wurdet und selbst nicht verstandet, (...)».

25. Pour Peter von Matt la trahison, "double, complémentaire", organise le texte entier. Liebesverrat. Die Treulosen in der Literatur, p. 244.

26. Ibid., p. 260 : « ..., der Unbekannten, die auf euren Hochzeiten den Klageruf anstimmt, auf nassen Füssen kommt und von deren Kuss ihr zu sterben fürchtet, so wie ihr zu sterben wünscht und nie mehr sterbt : ordnungslos, hingerissen und von höchster Vernunft. »

27. Ondine, p. 125.

28. Undine geht, p. 260 : «Mein Gedächtnis ist unmenschlich. An alles habe ich denken müssen, an jeden Verrat und jede Nichtigkeit. »

29. Ibid., p. 259 : «Wie könnte ich je an die Wichtigkeit eurer Verstrickungen glauben? Wie euch glauben, solange ich euch wirklich glaube, ganz und gar glaube, dass ihr mehr seid als eure schwachen, eitlen Äusserungen, eure schäbigen Handlungen, eure törichten Verdächtigungen. » 30. Lecouteux, S. Claude, Das Motiv der gestörten Mahrtenehe als Widerspiegelung der menschlichen Psyche. In : Vom Menschenbild im Märchen. Im Auftrag der Europäischen Märchengesellschaft, hrsg. von Janning, Jürgen, Gehrts, Heino u. Ossowski, Herbert. Röth, Kassel, 1980, p. 70.

31. Undine geht, p. 258 : «Aber ich habe euch mit einem Blick gelehrt, wenn alles vollkommen, hell und rasend war - ich habe euch gesagt : Es ist der Tod darin. Und : es ist die Zeit daran. Und zugleich : Geh Tod! Und : Steh still Zeit!».

32. Ingeborg Bachmann, Die Wahrheit ist dem Menschen zumutbar. Rede zur Verleihung des Hörspielpreises der Kriegsblinden, 17. März 1959. Dans : Ingeborg Bachmann, Werke, Band IV, Hrsg. Christine Koschel, Inge von Weidenbaum und Clemens Münster, München, Zürich, Piper \& Co. Verlag, 1978, p. 276 : «Es ist auch mir gewiss, dass wir in der Ordnung bleiben müssen, dass es den Austritt aus der Gesellschaft nicht gibt und wir uns aneinander prüfen müssen. Innerhalb dieser Grenzen aber haben wir den Blick gerichtet auf das Vollkommene, das Unmögliche, Unerreichbare, sei es in der Liebe, der Freiheit oder jeder reinen Grösse. »

33. Nous voyons en cette conception un parallèle intéressant avec une réflexion d'Elias Canetti qui, dans son récit autobiographique, rend compte de son admiration pour les tableaux de Breughel auxquels ils attribue une fonction similaire.

Elias Canetti, Le flambeau à l'oreille. Histoire d'une vie 1921-1931. Traduit par Michel-François Demet, Paris, Albin Michel, 1998, p. 440 : «Une des voies qui mènent à la réalité passe en effet par des tableaux. (...) L'expérience se tient alors tranquille, il la regarde dans les yeux. Il s'apaise dans la connaissance de cette réalité qui est la sienne propre, même si, ici, elle est peinte à son intention. En apparence, elle existerait aussi sans lui, mais cette apparence est trompeuse, le tableau a besoin de son expérience à lui pour s'éveiller. Elias Canetti, Die Fackel im Ohr. Lebensgeschichte 1921-1931, München, Hanser, 1993, p. 110 : « Denn ein Weg zur Wirklichkeit geht über Bilder. (...) Da hält die Erfahrung still, da sieht er ihr ins Gesicht. Da beruhigt er sich an der Kenntnis der Wirklichkeit, die seine eigene ist, obwohl sie ihm hier vorgebildet wurde. Scheinbar wäre sie auch ohne ihn da, doch dieser Anschein trügt, das Bild braucht seine Erfahrung, um zu erwachen.»

34. C'est l'interprétation que propose entre autres Françoise Rétif, Simone de Beauvoir et Ingeborg Bachmann. Tristan ou l'androgyne? La femme et son amour, Berne, Francfort s/M, New York, Paris, 
Lang 1989 (Publications universitaires européennes, Série 18, littérature comparée, vol. 51), p. $111 \mathrm{~s}$.

35. Undine geht, p. 254 : «Die nasse Grenze zwischen mir und mir. »

36. Dagmar Kann-Coomann, «Eine geheime langsame Feier»: Zeit und ästhetische Erfahrung im Werk Ingeborg Bachmanns, Frankfurt a./M., Bern, New York, Paris, Lang 1988. Europäische Hochschulschriften, Reihe 1, Deutsche Sprache und Literatur, Band 1070, p. 18.

37. Hans Christian Andersen, Eventyr, Fortalte for Born, Hrsg. E. Dal, Kopenhagen, 1963.

38. Undine geht, p. 253 : « (...), weil ich nicht aufhören kann, ihn zu rufen, Hans, Hans... » et p. 257 : «Ruf zum Ende. Zum Ende».

39. Ibid., p. 254 : «Guten Abend.»

«Guten Abend. »

«Wie weit ist es zu dir?»

"Weit ist es, weit. »

« Und weit ist es zu mir.»

40. C'est précisément le sujet de la nouvelle précédente de ce même recueil Ein Wildermuth (La vérité).

41. Ibid., p. 259 : «Dann sind alle Wasser über die Ufer getreten, die Flüsse haben sich erhoben, die Seerosen sind gleich hundertweis erblüht und ertrunken, und das Meer war ein machtvoller Seufzer, es schlug, schlug und rannte und rollte gegen die Erde an, dass seine Lefzen trieften von weissem Schaum. » L'allitération dans la phrase allemande se perd dans la traduction française qui par ailleurs ne respecte pas le lien existant entre « centaines » et « nénuphars ».

42. Ibid., p. 258 : «Wir liebten einander. Wir waren vom gleichen Geist. » et p. 257 : « Ich verstehe dich nicht, verstehe nicht, kann nicht verstehen!»

43. Ibid., p. 257 : «Ich habe die Konferenzen so vollkommen verstanden, eure Drohungen, Beweisführungen, Verschanzungen, dass sie nicht mehr zu verstehen waren. »

44. Dans le texte allemand, on lit: «Beinahe wahr. Beinahe mörderisch wahr.» La traduction française proposée ne nous paraît pas restituer le sens exact de ces termes: «Presque prophétiques. Meurtriers. »

45. Ibid., p. 263 : «Beinahe verstummt,

beinahe noch

den Ruf

hörend.

Komm. Nur einmal.

Komm.

46. Leçons de Francfort, p. 28.

Frankfurter Vorlesungen, p. 196 : « zu repräsentieren, seine Zeit zu repräsentieren, und etwas zu präsentieren, für das die Zeit noch nicht gekommen ist. »

\section{RÉSUMÉS}

Nouvelle sous forme d'une longue plainte adressée aux hommes, Undine geht (L'Adieu de la sirène Ondine) d'Ingeborg Bachmann reprend la thématique de l'impossible relation amoureuse entre Hans, représentant du genre humain et Undine, allégorie aux visages multiples, projection des désirs secrets de l'homme, symbole d'une existence utopique. 
En examinant les emprunts faits par ce texte publié en 1961 à des modèles littéraires préexistants, la présente étude tente de dégager la problématique essentielle de ce monologue douloureux témoignant de la quête désespérée d'un langage nouveau qui serait en mesure d'établir une véritable communication entre les êtres.

Oscillant constamment entre le passé et le présent, jouant sur l'identité insaisissable des deux personnages principaux, la nouvelle se caractérise par une tension sous-jacente presque insoutenable. Déplorant la fin d'une union, l'adieu de la sirène Undine ne semble néanmoins pas définitif. Si la séparation de Hans et d'Undine reste inéluctable, elle n'en demeure pas moins temporaire. Les paroles finales - un appel insistant lancé à l'autre - laissent croire en la possibilité d'une nouvelle rencontre : celle qui verra naître la création artistique.

In Form einer grossen Anklage, nimmt Ingeborg Bachmanns Novelle Undine geht erneut die Thematik einer unmöglichen Liebesbeziehung auf: der zwischen Hans, dem Stellvertreter des menschlichen Geschlechtes, und Undine, der facettenreichen Allegorie, Spiegelbild der geheimen Wünsche des Menschen, Sinnbild einer utopischen Existenz.

Unter Bezugnahme auf die Anlehnung dieses 1961 entstandenen Textes an bekannte literarische Modelle, versucht die vorliegende Untersuchung, die eigentliche Problematik dieses schmerzlichen Monologes aufzuzeigen. Letzterer zeugt von der verzweifelten Suche nach einer neuen Sprache, die es erlauben könnte, zu einer wirklichen Verständigung zwischen den Menschen zu gelangen.

Die Novelle, die beständig zwischen Vergangenheit und Gegenwart schwankt und mit der nicht eindeutigen Identität der beiden Hauptpersonen spielt, ist geprägt von einer unterschwelligen Spannung, die unerträglich zu werden droht. Wenn er auch das Ende einer Beziehung beklagt, so scheint der Abschied der Wasserfrau Undine doch nicht endgültig zu sein. Wenn die Trennung des Paares Undine und Hans auch unvermeidlich ist, so bleibt sie zeitlich beschränkt. Die letzten Worte - ein inständiges Rufen des anderen - lassen die Möglichkeit einer neuen Begegnung aufscheinen : jener Begegnung, die zur Entstehung des Kunstwerks führt.

\section{AUTEUR}

\section{MARION DUFRESNE}

Université Charles-de-Gaulle - Lille 3 